\title{
L'éducation thérapeutique et le patient douloureux chronique
}

\author{
Therapeutic Education and Patients with Chronic Pain
}

\author{
E. Collin \\ (C) Lavoisier SAS 2017
}

La médecine de la douleur est une médecine globale qui se bat depuis des années pour faire reconnaître sa particularité, très liée à son champ d'expertise. La douleur qu'elle soit aiguë ou chronique est « subjective », et la qualité de sa perception par un sujet donné au moment où cette douleur surgit va dépendre du contexte, de l'attention qui y est portée, de l'interprétation que le sujet en fait (souvent fonction de ses croyances), de son état émotionnel, de son humeur, de son état général (et possiblement de son patrimoine génétique) mais également de sa biographie et des expériences corporelles antérieures... La chronicisation de la douleur, au-delà de trois mois (en dehors d'une association à une maladie évolutive), résulte de facteurs pouvant être regroupés en trois catégories : une inhérente au sujet (déclinaison de ce qui vient d'être dit, état émotionnel...) et de son interaction avec l'entourage (affectif, social [travail...]) ; la deuxième correspond au parcours de soins y compris des acteurs de santé rencontrés (douleur initiale insuffisamment soulagée, communication défaillante des soignants, incohérence du parcours et du discours) et enfin du contexte social (harcèlement, accident de travail, licenciement, sentiment d'injustice...). La « prise en charge » globale des patients suivis dans des structures douleur spécialisées découle de cette complexité. Il faut débrouiller la situation et faire une évaluation des patients et de leur douleur « biopsychosociale » pour leur proposer « un programme multimodal » leur permettant de retrouver un sens à leur vie en dépit de leur douleur chronique. Pour cela, nous écoutons, nous informons, nous expliquons, nous faisons faire, nous leur apprenons à repérer, à anticiper leur douleur pour mieux prendre leurs traitements (médicamenteux ou non). Nous prenons du temps, ils voient plusieurs professionnels (médecins, infirmier, psychologue, kinésithérapeutes...), nous leur apprenons des techniques (TENS, relaxation...). Nous, professionnels entraînés à la prise en charge de patients

\footnotetext{
E. Collin $(\bowtie)$

Consultation d'étude et de traitement de la douleur, hôpital Avicenne, GH HUPSSD,

125, rue de Stalingrad, F-93000 Bobigny, France

e-mail : elisabeth.collin@upmc.fr
}

douloureux, faisons-nous de l'éducation thérapeutique ? peut-être, mais pourtant, si nous nous arrêtons là, trop souvent, les patients continuent à être arrêtés par leur douleur, à ne pas s'approprier les « savoirs », les compétences qu'on souhaite leur donner.

L'éducation thérapeutique, nous dit André Grimaldi dans ce numéro : «c'est un transfert de savoirs, une aide à la résilience et une pratique de la codécision. Ce n'est pas une prestation supplémentaire s'ajoutant à nos pratiques. $C$ 'est un autre rapport au temps, un autre rapport médecin/malade, un autre rapport médecin/paramédicaux. C'est la troisième médecine qui permet de prendre en charge les pathologies chroniques. \ Si l'on écoute Assal [1], père de l'éducation thérapeutique : «pour le soignant, améliorer des soins aux personnes souffrant d'une maladie chronique, c'est se diriger selon quatre axes :

- vouloir vraiment partager : une garantie de pouvoir se décentrer sur le malade ;

- être créatif afin de se protéger de la routine ;

- maîtriser son propre pouvoir de soignant afin de laisser le plus d'espace possible au malade ;

- suivre une logique pédagogique pour avancer pas à pas. Cela aidera le malade à maîtriser progressivement son autonomie thérapeutique. »»

Ainsi, l'éducation thérapeutique doit commencer par la formation du soignant [2]. Giordan et Golay et al. [3,4] démontrent de façon brillante que faire apprendre n'est pas enseigner, dire ou montrer, conditionner ou rendre actif. Cela ne correspond pas non plus à l'introduction de pédagogies actives ou de recettes pédagogiques. Apprendre est un processus paradoxal, voire conflictuel pour le patient. Il n'apprend que s'il est concerné, y trouve du plaisir, peut s'appuyer sur ses conceptions, mais peut aussi en lâcher, s'il a confiance (en soi, à la situation, aux soignants), trouve des aides, peut se confronter aux autres, prendre du recul. Il ne doit pas y avoir de recette, il faut juste s'adapter au patient. C'est justement le fondement même et l'intérêt de l'éducation thérapeutique qui permettent un changement de posture des soignants et du coup celui des patients. Ils vont ainsi 
pouvoir se mobiliser, s'approprier des compétences utiles pour s'adapter à la pathologie chronique. C'est cette thématique qui est abordée dans l'article cosigné avec Anne Coutaux dans ce numéro.

Gérard Reach attire notre attention sur l'éthique de l'éducation thérapeutique et les risques réels de manipulation nécessitant de proscrire toute tromperie intentionnelle et de bien faire la différence avec la persuasion qui laisse au patient la liberté d'effectuer ses choix.

Après cette «première » partie de réflexion sur l'éducation thérapeutique, ce dossier apporte des éléments sur des programmes pour tous patients douloureux chroniques, leurs modes d'organisations et sur leur mise en place. Grisell Vargas et collaboratrices nous font part de leur expérience canadienne dans la création d'un programme d'éducation thérapeutique pour tous patients douloureux chroniques et de l'intérêt (et la difficulté) de l'évaluation des programmes. Véronique Vion et collaborateurs présentent un outil magnifiquement coconstruit par une équipe pluriprofessionnelle et des patients, travail collaboratif au sein d'une région. Esther Soyeux et collaboratrices du réseau Lutter contre la douleur décrivent une organisation subtile du circuit éducatif au sein $\mathrm{du}$ réseau. Ce circuit permet une offre et un accès en ville d'ateliers éducatifs et d'apprentissage de techniques psychocorporelles variées, adaptées aux besoins des patients douloureux chroniques.

Dans une troisième partie, Martine Chauvin nous fait part de son parcours particulièrement riche mais nécessaire pour devenir patient expert. Des patients, que je remercie ici, témoignent de l'apport de l'éducation thérapeutique dans leur parcours de soins.

\section{Références}

1. Assal JP (2002) Et si une certaine pédagogie conduisait à une médecine plus globale ? Rev Med Suisse 2:22448

2. JO (2015) Cahier des charges des programmes d'éducation thérapeutique du patient et à la composition du dossier de demande de leur autorisation et de leur renouvellement. https://www.legifrance. gouv.fr/eli/arrete/2015/1/14/AFSP1501146A/jo/texte

3. Giordan A (2002) Apprendre ! Belin, Paris

4. Golay A, Lagger G, Giordan A (2009) Comment motiver le patient à changer. Maloine, Paris 